\title{
A THEORY OF INTERVAL ITERATION
}

\author{
L. B. RALL ${ }^{1}$
}

\begin{abstract}
A theory of interval iteration, based on a few simple assumptions, is given for the fixed point problem for operators in partially ordered topological spaces. A comparison of interval with ordinary iteration is made which shows that their properties are converse in a certain sense with respect to existence or nonexistence of fixed points. The theory of interval iteration is shown to hold without modification if the computation is restricted to a finite set of points, as in actual practice. In this latter case, interval iteration is shown to converge or diverge in a finite number of steps, for which an upper bound is given. By the introduction of a suitable iteration operator, the method of interval iteration is extended to the problem of solution of equations in linear spaces.
\end{abstract}

1. A fixed point problem. Suppose that $\phi$ is an operator which maps a partially ordered topological space $S$ into itself. A fixed point $y^{*} \in S$ of $\phi$ satisfies the equation

$$
y=\phi(y)
$$

solving this equation is called the fixed point problem for $\phi$ in $S$.

The partial ordering relation in $S$ will be denoted, as usual, by “ $\leqslant$ ". Elements $y$, $\bar{y}$ of $S$, such that $\underline{y} \leqslant \bar{y}$, define an interval $Y=[\underline{y}, \bar{y}]$ in $S$, which is the nonempty set

$$
Y=[\underline{y}, \bar{y}]=\{y \mid \underline{y} \leqslant y \leqslant \bar{y}, y \in S\} .
$$

The elements $y, \bar{y}$ are called, respectively, the lower and upper endpoints of $Y$. The set of all intervals in $S$ is denoted by $I S$. The elements $y$ of $S$ are identified with the corresponding degenerate intervals $y=[y, y]$ which have equal endpoints. It is assumed that the intersection of intervals is either an interval or the empty set $\varnothing$; this will hold if $S$ is a complete lattice [2].

The following assumptions will be made concerning the topology of $S$ :

(i) Intervals are closed subsets of $S$.

(ii) Each nondegenerate interval contains a limit point of countable order.

The last assumption means that if $Y$ is nondegenerate, then it contains a limit point $l$ such that each neighborhood of $l$ contains at least a countable number of points of $Y$ different from $l$. This technical property is called $\aleph_{0}$-compactness by Sierpiński [14].

Received by the editors December 28, 1981 and, in revised form, May 10, 1982. Presented April 16, 1982, to the 794th Meeting of the American Mathematical Society at Madison, Wisconsin.

1980 Mathematics Subject Classification. Primary 65J10, 65J15, 65G10.

Key words and phrases. Fixed point problems, interval iteration, convergence and divergence, existence and nonexistence of solutions, lower and upper bounds, finite convergence, solution of equations.

'Sponsored by the U.S. Army under Contract No. DAAG29-80-C-0041. 
2. Interval extensions and interval iteration. An operator which maps the set $I S$ of intervals in $S$ into itself is called an interval operator in $S$. An interval operator $\Phi$ is said to be an interval extension of an operator $\phi$ in $S$ if (i) it is an extension in the sense that

$$
\{\phi(y) \mid y \in Y\} \subset \Phi(Y), \quad Y \in I S,
$$

and (ii) $\Phi$ is inclusion monotone, that is,

$$
Y \subset Z \Rightarrow \Phi(Y) \subset \Phi(Z), \quad Y, Z \in I S .
$$

$\Phi$ is called an interval extension of an interval operator $F$ if $F(Y) \subset \Phi(Y)$ for $Y \in I S$ and (2.2) holds.

Definition 2.1. Given an initial interval $Y_{0}$ and an interval operator $\Phi$, the sequence $\left\{Y_{n}\right\}$ defined by

$$
Y_{n+1}=Y_{n} \cap \Phi\left(Y_{n}\right), \quad n=0,1,2, \ldots,
$$

is said to be generated by interval iteration starting from $Y_{0}$.

Note that, from (2.3),

$$
Y_{0} \supset Y_{1} \supset Y_{2} \supset \cdots \text {; }
$$

hence, interval iteration generates a nested (or descending) sequence of closed sets, and is thus a type of monotone iteration $[11,12]$.

DEFINITION 2.2. The interval iteration process (2.3) is said to diverge if

$$
Y_{N}=\varnothing \quad \text { (empty) }
$$

for some positive integer $N$; otherwise,

$$
Y^{*}=\bigcap_{n=0}^{\infty} Y_{n}
$$

is nonempty by the Cantor theorem [14, pp. 34-35], and the interval iteration is said to converge to the limit $Y^{*}=\lim _{n \rightarrow \infty}\left\{Y_{n}\right\}$ given by (2.6).

Thus, according to this definition, convergence and divergence of interval iteration have a converse relationship, as one would expect.

THEOREM 2.1. If $\Phi$ is an interval extension of the operator $\phi$ in $S$, and the initial interval $Y_{0}$ contains a fixed point $y^{*}$ of $\phi$, then the interval iteration (2.3) converges; furthermore,

$$
y^{*} \in Y^{*}=\lim _{n \rightarrow \infty}\left\{Y_{n}\right\}=\bigcap_{n=0}^{\infty} Y_{n} .
$$

Proof. This follows, as is well known [5, 8] from (2.3) and mathematical induction, since $y^{*} \in Y_{k} \Rightarrow \phi\left(y^{*}\right)=y^{*} \in \Phi\left(Y_{k}\right)$, and thus $y^{*} \in Y_{k+1}=Y_{k} \cap$ $\Phi\left(Y_{k}\right) \neq \varnothing$. Q.E.D.

The contrapositive of Theorem 2.1 is the following result, which is more incisive:

THEOREM 2.2. If $\Phi$ is an interval extension of $\phi$, and the interval iteration (2.3) diverges, then the initial interval $Y_{0}$ contains no fixed points $y^{*}$ of $\phi$.

This assertion was noted by Nickel [10] in connection with an interval version of Newton's method. 
Observe that convergence of interval iteration is a necessary, not sufficient, condition for the existence of a fixed point $y^{*} \in Y_{0}$ of $\phi$; divergence, on the other hand, is a sufficient condition for nonexistence of fixed points of $\phi$ in $Y_{0}$.

3. Comparison with ordinary iteration. The ordinary iteration method

$$
y_{n+1}=\phi\left(y_{n}\right), \quad n=0,1,2, \ldots,
$$

is often used to attempt to generate a sequence $\left\{y_{n}\right\}$ which converges to a fixed point $y^{*}$ of $\phi$, starting from some initial point $y_{0}$. If $\phi$ is continuous in the topology for $S$, in which also $\lim _{n \rightarrow \infty}\left\{y_{n}\right\}=y^{*} \in S$, then $y^{*}$ will be a fixed point of $\phi$, and if $Y_{0}$ is a closed subset of $S$ such that $\left\{y_{n}\right\} \subset Y_{0}$, then $y^{*} \in Y_{0}$. On the other hand, if $Y_{0}$ is a subset of $S$ which does not contain a fixed point of the continuous operator $\phi$, then the sequence generated by the iteration (3.1) cannot converge to a point of $Y_{0}$. The first alternative will be called convergence into $Y_{0}$, and the second divergence from $Y_{0}$. On the basis of these definitions and the corresponding concepts for interval iteration given in Definition 2.2, a comparison of ordinary and interval iteration is shown in Figure 3.1.

\begin{tabular}{c|c} 
Ordinary Iteration $y_{n+1}=\phi\left(y_{n}\right)$ & Interval Iteration $Y_{n+1}=Y_{n} \cap \Phi\left(Y_{n}\right)$ \\
\hline \hline Convergence (into $\left.Y_{0}\right) \Rightarrow$ & Existence $\left(y^{*} \in Y_{0}\right) \Rightarrow$ \\
Existence $\left(y^{*} \in Y_{0}\right)$ & Convergence $\left(Y=\cap_{n=0}^{\infty} Y_{n} \neq \varnothing\right)$ \\
\hline Nonexistence $\left(y^{*} \notin Y_{0}\right) \Rightarrow$ & Divergence (some $\left.Y_{n}=\varnothing\right) \Rightarrow$ \\
Divergence (from $\left.Y_{0}\right)$ & Nonexistence $\left(y^{*} \notin Y_{0}\right)$ \\
\hline
\end{tabular}

FIGURE 3.1. Ordinary and interval iteration compared

Thus, interval iteration stands in a converse relationship to ordinary iteration under the above assumptions. It is worth noting that in metric spaces $S$, the convergence of the ordinary iteration process (3.1) often depends on being able to choose the initial point $y_{0}$ close to the fixed point $y^{*}$, and that some operators $\phi$ have fixed points $y^{*}$ which repel the iteration sequence $\left\{y_{n}\right\}$ for all $y_{0} \neq y^{*}$. The convergence of interval iteration, on the other hand, follows if the initial interval $Y_{0}$ is "large enough" to contain a fixed point $y^{*}$ of $\phi$.

4. Applications of interval iteration. Interval iteration may be applied in several ways to the fixed point problem (1.1).

$1^{\circ}$. Suppose that the interval $Y_{0}$ is known to contain a fixed point $y^{*}$ of $\phi$, perhaps on the basis of a nonconstructive fixed point theorem. In this case, the interval iteration (2.3) will converge, and may be used to obtain lower and upper bounds for $y^{*}$, namely,

$$
\underline{y}_{n} \leqslant y^{*} \leqslant \bar{y}_{n}, \quad n=0,1,2, \ldots,
$$

where $Y_{n}=\left[\underline{y}_{n}, \bar{y}_{n}\right]$, and in the limit,

$$
\underline{y}^{*} \leqslant y^{*} \leqslant \bar{y}^{*}
$$

where $Y^{*}=\left[\underline{y}^{*}, \bar{y}^{*}\right]$ is the limit (2.6) of $\left\{Y_{n}\right\}$. 
The bounds (4.1) give improved results as long as the inclusions (2.4) are strict. However, if $Y_{N+1}=Y_{N}$ for some positive integer $N$, then

$$
Y^{*}=\bigcap_{n=0}^{\infty} Y_{n}=Y_{N}
$$

this is called finite convergence of the interval iteration (2.3). If finite convergence takes place, then the best lower and upper bounds obtainable for $y^{*}$, starting from $Y_{0}=\left[\underline{y}_{0}, \bar{y}_{0}\right]$, are

$$
\underline{y}^{*}=\underline{y}_{N} \leqslant y^{*} \leqslant \bar{y}_{N}=\bar{y}^{*} .
$$

$2^{\circ}$. If it is not known whether or not $Y_{0}$ contains a fixed point $y^{*}$ of $\phi$, interval iteration may still be useful in one of the following ways:

(i) As long as the interval iteration is producing intervals which decrease at each step (strict inclusion holds in (2.4)), then an existence test that fails because $Y_{0}, Y_{1}, \ldots, Y_{N-1}$ are "too large" may succeed for $Y_{N}$. This $Y_{N}$ may then be taken as the initial interval $Y_{0}$, and one has the favorable case $1^{\circ}$ discussed above.

(ii) If the iteration produces an empty intersection (divergence), then this establishes conclusively that the initial interval $Y_{0}$ does not contain a fixed point $y^{*}$ of $\phi$, so that this interval may be excluded from further consideration.

There is, of course, a third possibility:

(iii) The interval iteration (2.3) leads only to an interval $Y^{\circ}$ in which no conclusive assertion about existence or nonexistence of a fixed point $y^{*}$ of $\phi$ is available. ( $Y^{\circ}$ may be the limit $Y^{*}$ if obtained in a finite number of steps, or otherwise.)

Possible alternatives in this situation include partition of the resulting interval $Y^{\circ}$ into subintervals for further examination, a strategy developed by Moore and Jones [9], or acceptance of $Y^{\circ}$ as a generalized or pseudosolution (relative to the initial interval $Y_{0}$ ) of the fixed point problem. This latter choice may be useful in the development of an interval version of regularization of solutions of ill-posed problems.

5. Interval iteration on a grid. The ordinary iteration process (3.1) is a poor model of what actually occurs in computation, since it is usually impossible to carry out the indicated transformations exactly. Interval iteration, on the other hand, is readily adaptable to actual machine computation, and its theory can be preserved intact.

Suppose that $G$ (called a grid) is a finite subset of the space $S$. Here, one may think of the set of numbers which have exact representations on a given computer, and finite Cartesian products of such a set. The subset of $I S$ consisting of intervals with endpoints in $G$ will be denoted by $I G$, that is,

$$
I G=\{[a, b] \mid a, b \in G\} .
$$

The union of all intervals in $I G$, considered as subsets of $S$, defines a closed subset $D$ of $S$, since $I G$ is a finite collection of closed sets [14]. The operation of directed rounding will now be defined in $I D$, the set of all intervals in $S$ having endpoints in D.

Definition 5.1. For $x \in D$, the upward rounding operator $\Delta$ to $G$ is defined by

$$
\Delta x=\min \{b \mid b \geqslant x, b \in G\},
$$


and the downward rounding operator $\nabla$ to $G$ by

$$
\nabla x=\max \{a \mid a \leqslant x, a \in G\} .
$$

For $X=[\underline{x}, \bar{x}] \in I D$, the directed rounding operator $\square$ to $I G$ is defined by

$$
\square X=\square[\underline{x}, \bar{x}]=[\nabla \underline{x}, \Delta \bar{x}], \quad X=[\underline{x}, \bar{x}] \in I D .
$$

It follows immediately from this definition that $\square$ is an inclusion monotone interval operator. Furthermore, if $\phi$ maps $D$ into itself and $\Phi$ is an interval extension of $\phi$, then $\square \Phi$ will be an interval extension of $\phi$ which maps $I G$ into $I G$. Thus, for actual computation, it may be assumed that the interval extension $\Phi$ of $\phi$ in (2.3) has been constructed to map $I G$ into itself. This means that the transformed intervals $\Phi\left(Y_{n}\right)$ will be exactly representable in terms of elements of $G$ for $Y_{n} \in I G$. The theory of interval iteration given above applies to operators of this type without modification. Furthermore, under the following reasonable assumption, the entire interval iteration (2.3) may be carried out exactly, using only elements of the grid $G$.

Assumption 5.1 (Intersection Property). If $X, Z \in I G$, then $X \cap Z=\varnothing$, the empty set, or $X \cap Z \in I G$.

Thus, if $I G$ has the intersection property, and the interval extension $\Phi$ of $\phi$ has been constructed to map $I G$ into itself, then the selection of an initial interval $Y_{0} \in I G$ will assure that the intervals $Y_{1}, Y_{2}, \ldots$ generated by the interval iteration (2.3) also belong to $I G$. Furthermore, this interval iteration on $I G$ will always converge or diverge in a finite number of steps. To see this, let $G_{\sharp} X=G_{\sharp}[\underline{x}, \bar{x}]$ denote the number of grid points (elements of $G$ ) contained in the interval $X \in I G$. Then the following result holds:

TheOREM 5.1. On IG, the interval iteration (2.3) will converge or diverge in at most $G \#\left(Y_{0}\right)$ steps.

Proof. If the interval iteration (2.3) has not terminated in $n$ steps by divergence or convergence, then it will have generated distinct intervals $Y_{0}, Y_{1}, \ldots, Y_{n} \in I G$, including the initial interval $Y_{0}$. Since (2.4) holds, one has

$$
G \sharp\left(Y_{n}\right) \leqslant G \sharp\left(Y_{n-1}\right)-1 \leqslant G \sharp\left(Y_{n-2}\right)-2 \leqslant \cdots \leqslant G \sharp\left(Y_{0}\right)-n \text {. }
$$

Thus, the maximum length of a sequence of distinct nested intervals in $I G$ is $\nu=G \sharp\left(Y_{0}\right)-1$, since each interval in $I G$ must contain at least one point of $G$. If the interval iteration has not terminated by the $\nu$ th step, then

$$
G \#\left(Y_{\nu}\right)=1
$$

by (5.5), which implies that $Y_{\nu}$ is a degenerate interval. Now one has either $Y_{\nu} \subset \Phi\left(Y_{\nu}\right)$, in which case $Y_{\nu+1}=Y_{\nu}$ (convergence in $\nu$ steps), or $Y_{\nu} \cap \Phi\left(Y_{\nu}\right)=\varnothing$ (divergence in $\nu+1=G \sharp\left(Y_{0}\right)$ steps). Q.E.D.

Thus, in actual computation, convergence or divergence of an interval iteration is an observable event in principle, since one works on a grid of machine numbers. Of course, $G \sharp\left(Y_{0}\right)$, although finite, could be prohibitively large; however, termination of interval iteration is usually abserved in far fewer steps. The construction of the interval operator $\Phi$ is crucial to the success of interval iteration [5], but depends heavily on the nature of $S, \phi$, and the grid $G$ available. 
6. Solution of equations. In many applications, $S$ is a linear space, and the problem of interest is to find a solution $x^{*}$ of the equation

$$
f(x)=0 \text {. }
$$

Interval methods for the solution of this problem have been developed by Alefeld [1], Krawczyk [4, 5], Moore [6, 8], Nickel [10], and others. One approach is to transform (6.1) into a fixed point problem (1.1) by the introduction of an iteration operator $\phi$. For example, one can take

$$
\phi(x)=x-Y f(x)
$$

to define $\phi$, where $Y$ is an invertible linear operator in $S$. Given an interval extension $F$ of $f$, the corresponding interval extension $\Phi$ of $\phi$ is

$$
\Phi(X)=X-Y F(X),
$$

using interval arithmetic $[6,8]$. If $S$ is a Banach space, and $f$ has a Fréchet derivative $f^{\prime}$, then

$$
\phi^{\prime}(x)=I-Y f^{\prime}(x),
$$

where $I$ denotes the identity operator, and a more accurate interval extension of $\phi$ can be constructed on the basis of its mean-value form [3]. Let $F^{\prime}$ be an interval extension of $f^{\prime}$. Then the corresponding interval extension $\Phi^{\prime}$ of $\phi^{\prime}$ is given by

$$
\Phi^{\prime}(X)=I-Y F^{\prime}(X),
$$

and the mean-value form of (6.2) is, for $y \in X$,

$$
\Phi(X)=y-Y f(y)+\left\{I-Y F^{\prime}(X)\right\}(X-y),
$$

and this interval extension $\Phi$ of $\phi$ is called the Krawczyk iteration operator. It arose from consideration of an interval version of Newton's method [4] and has many useful properties $[5,7,11]$. In actual practice, computation would be done with a rounded version of (6.6). Suppose that $F, F^{\prime}$ are interval extensions in $I G$ of $f, f^{\prime}$, then

$$
\Phi(X)=\square\left\{y-Y F(y)+\left\{I-Y F^{\prime}(X)\right\}(X-y)\right\}
$$

will have values in $I G$ for $y \in G, X \in I G$. An even more rounded interval extension of (6.6) to $I . G$ is

$$
\Phi(X)=\square\left\{\square\{y-\square Y F(y)\}+\square\left\{\square\left\{I-\square Y F^{\prime}(X)\right\} \cdot \square(X-y)\right\}\right\},
$$

which models a realistic computational interval operator.

For a simple example, consider the scalar case of (1.1) with $f(x)=x-x^{2}$. Here, an interval extension of $f(x)$ is simply $F(X)=X-X^{2}$, and for $X_{0}=\left[\frac{1}{8}, \frac{3}{8}\right]$; one has

$$
0 \in F\left(X_{0}\right)=\left[\frac{1}{8}, \frac{3}{8}\right]-\left[\frac{1}{8}, \frac{3}{8}\right]^{2}=\left[-\frac{1}{64}, \frac{13}{64}\right],
$$

so it is possible that $X_{0}$ contains a solution $x=x^{*}$ of (1.1) in this case. However, interval iteration using (6.6) with $Y=1, y=m(X)$, the midpoint of $X$, and the interval extension $F^{\prime}(X)=1-2 X$ gives

$$
X_{1}=\left[\frac{1}{8}, \frac{5}{32}\right], \quad \Phi\left(X_{1}\right)=\left[\frac{61}{4096}, \frac{101}{4096}\right], \quad X_{1} \cap \Phi\left(X_{1}\right)=\varnothing,
$$


which proves that $X_{0}=\left[\frac{1}{8}, \frac{3}{8}\right]$ contains no solutions of $x-x^{2}=0$. On the other hand, for $X_{0}=\left[-\frac{1}{4}, \frac{1}{4}\right]$, for example, the same interval iteration gives a sequence which converges rapidly to $X^{*}=[0,0]$; a degenerate interval which can be identified with the solution $x^{*}=0$ of the equation considered.

\section{REFERENCES}

1. G. Alefeld, Intervallanalytische Methoden bei nichtinearen Gleichungen, Jahrb. Überblicke Math. 1979 (1979), 63-78.

2. G. Birkhoff, Lattice theory, rev. ed., Amer. Math. Soc. Colloq. Publ., vol. 25, Amer. Math. Soc., Providence, R. I., 1948.

3. O. Caprani and K. Madsen, Mean value forms in interval analysis, Computing 25 (1980), 147-154.

4. R. Krawczyk, Newton-Algorithmen zur Bestimmung von Nullstellen mit Fehlerschranken, Computing 4 (1969), 187-201.

5. Interval extensions and interval iterations, Computing 24 (1980), 119-129.

6. R. E. Moore, Interval analysis, Prentice-Hall, Englewood Cliffs, N. J., 1966.

7. _ A test for existence of solutions to nonlinear systems, SIAM J. Numer. Anal. 14 (1977), $611-615$

8. __ Methods and applications of interval analysis, SIAM Studies in Appl. Math., vol. 2, SIAM, Philadelphia, Pa., 1979.

9. R. E. Moore and S. T. Jones, Safe starting regions for iterative methods, SIAM J. Numer. Anal. 14 (1977), 1051-1065.

10. K. Nickel, On the Newton method in interval analysis, MRC Tech. Summary Rept. No. 1136, Univ. of Wisconsin-Madison, 1971.

11. __ Stability and convergence of monotonic algorithms, J. Math. Anal. Appl. 54 (1976), 157-172.

12. J. M. Ortega and W. C. Rheinboldt, Iterative solution of nonlinear equations in several variables, Academic Press, New York, 1970.

13. L. B. Rall, A comparison of the existence theorems of Kantorovich and Moore, SIAM J. Numer. Anal. 17 (1980), 148-161.

14. W. Sierpiński, General topology (C. Cecilia Krieger, translator), Univ. of Toronto Press, Toronto, 1952.

Mathematics Research Center, University of Wisconsin-Madison, Madison, Wisconsin 53706 